\title{
Self-organization of (001) cubic crystal surfaces
}

\author{
Laurent Proville \\ Groupe de Physique des Solides, UMR 7588-CNRS \\ Universités Paris 7 \& Paris 6, Tour 23, 2 pl. Jussieu 75251, Paris Cedex 05, France
}

(November 13, 2018)

\begin{abstract}
Self-organization on crystal surface is studied as a two dimensional spinodal decomposition in presence of a surface stress. The elastic Green function is calculated for a (001) cubic crystal surface taking into account the crystal anisotropy.

Numerical calculations show that the phase separation is driven by the interplay between domain boundary energy and long range elastic interactions. At late stage of the phase separation process, a steady state appears with different nanometric patterns according to the surface coverage and the crystal elastic constants.
\end{abstract}

Typeset using REVTEX 


\section{INTRODUCTION}

Self-organization (SO) on solid surface is an efficient mean for growing nanostructures with regular sizes and spacings. The models proposed by Marchenko [1] and Vanderbilt et al. [2,3] are the basis of the theoretical framework to understand the SO phenomenon. They enhanced the interplay between the long range elastic interaction yielded by the underlying crystal and the domain boundary energy. Indeed, the former is minimum when two surface defaults are separated by a distance as large as possible while the latter is minimum when only one compact domain appears onto the surface. So when these two ingredients are present (see below for experimental descriptions), the surface ground state structure should balance the aforementioned interactions. The purpose of [1] 3] was to evaluate this structure in different cases.

Assuming that two phases, called A and B, coexisting onto a crystal surface, have different intrinsic stresses, the authors of Refs. [1,2] showed that a state which consists of stripes domains occupied alternatively by A and B lowers the energy with a period selection. This period depends on the crystal stress energy compared with the domain boundary energy and it varies exponentially with the ratio of those two quantities. In order to perform an analytical surface elastic Green function calculation, Marchenko and Vanderbilt both assumed either a crystal anisotropy along one direction of the surface or the anisotropy of the intrinsic stresses. Those works specially addressed the cases of corrugated crystal surfaces and crystal surface reconstruction with broken symmetry (see Ref. «, [5] for recent relevant experimental analysis).

Comparing the stability of different periodic domains in an isotropic 2-dimensional dipolar model, Vanderbilt et al. [3] proved that at intermediate coverage, $\theta_{0}>0.28$, the stripe structure is the optimal candidate for the 2-dimensional system ground state while at low coverage, $\theta_{0}<0.28$, the droplets structure is more stable. As the dipole-dipole interaction is similar to the elastic interaction, i.e., it decreases as the inverse of the distance to the power three, these Vanderbilt's results hold for the solid surface SO. It shows that the anisotropy of 
the intrinsic stress surface, assumed in Refs. [1,2] is not essential in the SO process since the patterning occures even if the surface is isotropic with no symmetry breaking. The studies of Refs. [1] emphasize the main physical ingredients which drive SO, i.e. , the interplay between domain boundary energy and elastic interaction energy.

Recent analysis of chemisorbed mono-layers on (001) Copper surfaces, via Scaning Tunneling Microscopy (STM) [6 8] and Spot Profile Analysing Low Energy Electron Diffraction (SPA-LEED) [9] show mesoscopic morphologies different from droplet or stripe structures. For the $\mathrm{N} / \mathrm{Cu}(001)$ case, the Nitrogen is chemically adsorbed on a (001) Copper crystal surface, and agregates within square-shaped islands that may arrange either in 1-dimensional rafts at low Nitrogen coverage or in a 2-dimensional array at intermediate coverages. The experimental works mentioned above [7,9] motivated the present study.

Here we propose to describe the SO kinetics on solid surface as a 2-dimensional spinodal decomposition. In this standard theory, we include the stress energy due to the underlying crystal and therefore the surface elastic Green function is calculated. The 2D Cahn-Hilliard equation which drives the surface diffusion, is integrated with computer means. Our method allows us to capture the SO kinetics together with the elastic anisotropy due to the crystal symmetries. The latter feature is proved to play a role in the nanometric arrangements. For simplicity, our study is focused on a (001) cubic crystal surface.

In the last decade, a similar theory has been developed under the name of "phase field" by Khachaturyan et al. for the kinetics of phase transition in alloys [10]. The phase field name is also used for models of solidification; see [11] for recent developments. We choose not to use this ambiguous terminology. In the framework of the SO spinodal theory, the implied coexistent phases may represent either two types of crystal facet, noted A and B or a chemical adsorbed layer A over a clean crystal surface B. In the latter case, we neglect the possible layer thickness.

Our results exhibit a steady state at late stage of the separation process which shows different mesoscopic patterns according to the coverage and to the elastic constants of the materials. Because of the crystal cubic symmetry, the nanometric morphologies of the final 
state may differ from the one predicted in Refs. [1] 3]. At low coverage, rafts of either disk or square shaped islands appear while at intermediate coverage a branched stripe structure occurs. The elastic constants of the material are shown to determine the preferential orientations of the rafts and of the stripes. If the surface square symmetry is broken, the kinetics final state is similar to those predicted in Refs. [1,2], i.e. , an assembly of regular spaced stripes.

\section{DOMAIN BOUNDARY ENERGY}

The covering parameter $\theta$ describes the A-B coexistent phases at the solid surface. These phases may differ by their composition or their geometry. Let say that $\theta=0$ for $\mathrm{B}$ and $\theta=1$ for A. If one assumes that the spatial surface variations of $\theta$ are smooth with respect to the atomic scale, a 2-dimensional coarse-graining procedure is thus relevant to represent the mesoscopic system state. If $d$ is the size of the elementary coarse-grained surfaces, we introduce the mesoscopic quantity $\theta(\mathbf{r})$ which is the $\theta$-average, performed over a whole elementary surface, centered at position $\mathbf{r}$. The $\theta(\mathbf{r})$ variable is the mesoscopic local coverage of the surface since it is the A quantity per unit area which is present in the $\mathbf{r}$ vicinity. The $\theta$-average over the entire surface is written $\theta_{0}$ and it is assumed to be conserved during the system time evolution.

An inhomogeneous mixing of $\mathrm{A}$ and $\mathrm{B}$ phases involves an energy increasing because of atomic bond breaking at domain boundaries. This energy together with the entropic term due to the surface inhomogeneity are capted in the 2-dimentional free Ginzburg-Landau (GL) functional energy:

$$
F_{\text {chem }}=F_{0} \cdot \iint_{S}\left\{\frac{\gamma}{2}\left[\left(\frac{\partial \theta}{\partial x_{1}}\right)^{2}+\left(\frac{\partial \theta}{\partial x_{2}}\right)^{2}\right]+\hat{f}(\theta)\right\} d \mathbf{r}
$$

We introduce here the adimensional free energy density $\hat{f}=16 . \theta^{2}(1-\theta)^{2}$ which is a double well potential with minima for $\theta=0$ and $\theta=1$. The hat notation points out the adimen-

tional quantities. The $\hat{f}$ form has no direct influence on the mesoscopic structure providing 
GL functional is invariant with respect to the (001) surface space group. Let note $\hat{g}(\theta)$, the continuous Hamiltonian including both the 2D gradient term and the free energy density $\hat{f}$.

The $F_{0}$ and $\gamma$ scalars are respectively the free energy density constant and the amplitude of the gradient term that both must be adjusted to set the model domain boundary energy $I$ to a realistic value, i.e., around $10 \mathrm{meV} / \AA$ (see Ref. [2]). At phase equilibrium, the I

quantity is given by $I=F_{0} \int_{B}^{A} \hat{g}(\theta) d \vec{l}$ where the integration is performed along a line path that goes from inside a B phase domain to inside an A domain [12]. It is easy to see that the previous path integral is overvalued by the product of the A-B interface width times the $\hat{f}$ maximum value, i.e., $\hat{f}(\theta=0.5)=1$. Fixing $\gamma=20 d^{2}$, which insure the $\theta(\mathbf{r})$ space variation smoothness, the A-B interface width is then around $5 . d$, at equilibrium. Therefore, to obtain $I \approx 10 \mathrm{meV} / \AA$, $F_{0} . d$ must be fixed to $2 \mathrm{meV} / \AA$ (or $3.210^{-12} \mathrm{~J} / \mathrm{m}$ ). As our investigations are focused over the nanometer scale, we choose to set $d=1 \mathrm{~nm}$ and thus $F_{0}=3.2 \mathrm{~mJ} / \mathrm{m}^{2}$. To study larger space scales as it would be suitable for Silicon which may exhibit a $100 \mathrm{~nm}$ vicinal period [2], then it is sufficient to increase the $d$ parameter and to adjust subsequently $F_{0}$, eventually changing the domain boundary energy $I$ if necessary.

For simplicity, we choose to neglect the possible $\gamma$ variations with respect to the crystal surface direction. Such a feature may be yielded from a crystal step anisotropy but here our study is focused on the elastic anisotropy due to the crystal symmetries.

\section{SURFACE GREEN FUNCTION}

An important stage of this work consists in the calculation of the cubic crystal surface elastic Green function. Let note $\mathbf{P}(\mathbf{r})$ a surface external force at position $\mathbf{r}$. In cartesian coordinates, the (001) surface is defined by $\left(x_{3}=0\right)$ and $\mathbf{r}=\left(x_{1}, x_{2}\right)$. The semi-infinite crystal occupies the half space $x_{3} \geq 0$. The surface normal is the $\mathbf{n}$ unit vector. The mechanic equilibrium condition at the surface is given by:

$$
\sigma_{i, j}\left(\mathrm{r}, x_{3}=0\right) . n_{j}=P_{i}(\mathrm{r})
$$


where $n_{j}$ is a $\mathbf{n}$ component and the summation over subscript $j$ is implicit. The crystal bulk stress, $\sigma_{i, j}\left(\mathrm{r}, x_{3}\right)$ is due to the crystal displacements and these quantities are related to each other by the Hooke law: $\sigma_{i, j}=\lambda_{i, j, k, l} \partial u_{k} / \partial x_{l}$. The forth order tensor $\lambda_{i, j, k, l}$ gives the crystal elastic constants and for a cubic crystal symmetry, this tensor is composed with three non zero coefficients [14], namely $\lambda_{i, i, i, i}=C_{11}, \lambda_{i, i, j, j}=C_{12}$ and $\lambda_{i, j, i, j}=\lambda_{i, j, j, i}=C_{44}$. In case of copper, those coefficients are : $C_{11}=1.68310^{11} \mathrm{~J} / \mathrm{m}^{3}, C_{12}=1.22110^{11} \mathrm{~J} / \mathrm{m}^{3}$ and $C_{44}=0.75710^{11} \mathrm{~J} / \mathrm{m}^{3}$ (see Physics Handbooks).

At mechanic equilibrium, the bulk displacements fulfill the Lamé equation:

$$
\lambda_{i, j, k, l} \frac{\partial^{2} u_{k}}{\partial x_{j} \partial x_{l}}=0
$$

Except constants, the displacement functions are fully determined by the set of equations (3.1,3.2). As proposed in Ref. [15] for the isotropic case, these equations may be solved by writing the displacements as 2-dimensional Fourier transforms:

$$
u_{i}\left(\mathbf{r}, x_{3}\right)=\iint \exp (i \mathbf{Q} \cdot \mathbf{r}) \cdot \tilde{u}_{i}\left(\mathbf{Q}, x_{3}\right) d \mathbf{Q}
$$

Note that the Fourier components $\tilde{u}_{i}\left(\mathbf{Q}, x_{3}\right)$ depend on both the wave vector $\mathbf{Q}=\left(q_{1}, q_{2}\right)$ and $x_{3}$. Once the set of Eqs. (3.1.3.2) is expressed using Eq.(3.3), the subsequent differential equations involving $\tilde{u}_{i}$ and their derivatives with respect to $x_{3}$ turn out to be linear, so that $\tilde{u}_{i}\left(\mathbf{Q} \neq 0, x_{3}\right)$ have an exponential dependence on the bulk penetration length $x_{3}$ :

$$
\tilde{u}_{j}\left(\mathbf{Q} \neq 0, x_{3}\right)=\sum_{l} \beta_{j, l} \exp \left(-\alpha_{l} . x_{3}\right)
$$

where both $\beta_{j, l}$ and $\alpha_{l}$ depend on the wave vector $\mathbf{Q}$. As the whole sample is at rest, the $\mathbf{P}$ average is assumed to be zero which implies $\tilde{u}_{j}\left(\mathbf{0}, x_{3}\right)=0$. The Eq. (3.2) is then reduced to a linear equation $M\left(\mathbf{Q}, \alpha_{l}\right)\left(\beta_{j, l}\right)=0$ where $M\left(\mathbf{Q}, \alpha_{l}\right)$ is the $3 \times 3$ matrix:

$$
M\left(\mathbf{Q}, \alpha_{l}\right)=\left(\begin{array}{ccc}
C_{11} q_{1}^{2}+C_{44} q_{2}^{2}-C_{44} \alpha_{l}^{2} & \left(C_{12}+C_{44}\right) q_{1} q_{2} & \left(C_{12}+C_{44}\right) i q_{1} \alpha_{l} \\
\left(C_{12}+C_{44}\right) q_{1} q_{2} & C_{11} q_{2}^{2}+C_{44} q_{1}^{2}-C_{44} \alpha_{l}^{2} & \left(C_{12}+C_{44}\right) i q_{2} \alpha_{l} \\
-\left(C_{12}+C_{44}\right) i q_{1} \alpha_{l} & -\left(C_{12}+C_{44}\right) i q_{2} \alpha_{l} & C_{11} \alpha_{l}^{2}-C_{44}\left(q_{1}^{2}+q_{2}^{2}\right)
\end{array}\right)
$$


As usual, the non-trivial solutions are such as $\operatorname{det}\left(M\left(\mathbf{Q}, \alpha_{l}\right)\right)=0$. This yields a third degree polynomial equation for the $\alpha_{l}^{2}$ parameters that we solve numerically. The only three $\alpha_{l}$ values with positive real parts are physically acceptable. At least one of the $\alpha_{l}$ is real, the two other roots may be either real or complex conjugated depending on the sign of the $\chi$ parameter:

$$
\chi=C_{11}-C_{12}-2 C_{44}
$$

This combination of the elastic constants is related to the elastic anisotropy of the cubic crystal (see Ref. [14]). When $\chi=0$, the crystal is isotropic and the three $\alpha_{j}$ are degenerate. This case was addressed in Refs. 14, 15. One retains as examples that the Copper and Gold $\chi$ 's are negative $\left(\chi_{C u}=-1.0, \chi_{A u}=-0.5\right)$ and the Chromium and Niobium $\chi^{\prime}$ 's are positive $\left(\chi_{C r}=+1.8, \chi_{N b}=+0.5\right)$.

To each $\alpha_{j}$ corresponds a unique set of three coefficients $\left(\beta_{j, l}\right)$ that are determined by inverting Eq. (3.1). Noting $\tilde{P}_{j}$ the Fourier transform of the force component $P_{j}$, we write the Fourier counterpart of the Eq. (3.1):

$$
\begin{aligned}
\sum_{l=(1,2,3)}-\alpha_{l} \beta_{l, 1}+i q_{1} \beta_{l, 3} & =\tilde{P}_{1} \\
\sum_{l=(1,2,3)}-\alpha_{l} \beta_{l, 2}+i q_{2} \beta_{l, 3} & =\tilde{P}_{2} \\
\sum_{l=(1,2,3)} C_{12}\left(i q_{1} \beta_{l, 1}+i q_{2} \beta_{l, 2}\right)-C_{11} \alpha_{l} \beta_{l, 3} & =\tilde{P}_{3}
\end{aligned}
$$

Combining the first and second rows with the third rows of the matrix $M\left(\mathbf{Q}, \alpha_{l}\right)$ (see Eq. (3.5)), one easily gets both equations

$$
\begin{gathered}
\beta_{l, 1}=i q_{1} \cdot \beta_{l, 3} \Gamma_{l, 1} / \alpha_{l} \\
\beta_{l, 2}=i q_{2} \cdot \beta_{l, 3} \Gamma_{l, 2} / \alpha_{l}
\end{gathered}
$$

where we write: 


$$
\begin{aligned}
\Gamma_{l, 1} & =\frac{\left(C_{11}-C_{12}-C_{44}\right) \alpha_{l}^{2}-C_{44}\left(q_{1}^{2}+q_{2}^{2}\right)}{\left(C_{11}-C_{12}-C_{44}\right) q_{1}^{2}+C_{44}\left(q_{2}^{2}-\alpha_{l}^{2}\right)} \\
\Gamma_{l, 2}= & \frac{\left(C_{11}-C_{12}-C_{44}\right) \alpha_{l}^{2}-C_{44}\left(q_{1}^{2}+q_{2}^{2}\right)}{\left(C_{11}-C_{12}-C_{44}\right) q_{2}^{2}+C_{44}\left(q_{1}^{2}-\alpha_{l}^{2}\right)}
\end{aligned}
$$

provided the denominators of $\Gamma_{i, 1}$ and $\Gamma_{i, 2}$ are non zero. If not, which occures for either $q_{1}=0$ or $q_{2}=0$ then it is easy to show that $\beta_{l, 3}=0$ and the calculation can be performed as well. In addition let us note

$$
\Gamma_{l, 3}=-C_{11} \alpha_{l}-C_{12}\left(q_{1}^{2} \Gamma_{l, 1}+q_{2}^{2} \Gamma_{l, 2}\right) / \alpha_{l}
$$

We rewrite Eq. (3.7) as a linear equation $N_{j, l} \beta_{l, 3}=\tilde{P}_{j}$ where the $3 \times 3$ matrix $N$ is defined as follows:

$$
N(\mathbf{Q})=\left(\begin{array}{ccc}
i q_{1}\left(1-\Gamma_{1,1}\right) & i q_{1}\left(1-\Gamma_{2,1}\right) & i q_{1}\left(1-\Gamma_{3,1}\right) \\
i q_{2}\left(1-\Gamma_{1,2}\right) & i q_{2}\left(1-\Gamma_{2,2}\right) & i q_{2}\left(1-\Gamma_{3,2}\right) \\
\Gamma_{1,3} & \Gamma_{2,3} & \Gamma_{3,3}
\end{array}\right)
$$

Provided $q_{1} \neq q_{2}$, this matrix (3.11) can be inverted which gives the $\beta_{l, 3}$ 's as linear functions of the $P_{j}(\mathbf{r})$ Fourier transforms : $\beta_{l, 3}=N_{l, j}^{-1} \tilde{P}_{j}$. One must distinguish the case $q_{1}=q_{2}$ for which details are not presented but are simple to deal with. Using Eqs. (3.8), one gets the whole set of $\beta_{l, j}$ 's and thus $\tilde{u}_{k}\left(\mathbf{Q}, x_{3}=0\right)=G_{k, j}(\mathbf{Q}) \tilde{P}_{j}(\mathbf{Q})$ where $G_{k, j}$ is the surface elastic Green function that we write as follows:

$$
\begin{aligned}
G_{1, j}(\mathbf{Q}) & =i q_{1} \sum_{l} N_{l, j}^{-1} \Gamma_{l, 1} / \alpha_{l} \\
G_{2, j}(\mathbf{Q}) & =i q_{2} \sum_{l} N_{l, j}^{-1} \Gamma_{l, 2} / \alpha_{l} \\
G_{3, j}(\mathbf{Q}) & =\sum_{l} N_{l, j}^{-1}
\end{aligned}
$$

The latest function varies as $1 /|\mathbf{Q}|$. The total elastic energy of the system is given at mechanical equilibrium according to Eqs. (3.1, 3.2) by : 


$$
E_{e l}=-1 / 2 \int_{x_{3}=0} P_{i} \cdot u_{i} d S
$$

that gives an analytical expression of $E_{e l}$ in the Fourier space:

$$
E_{e l}=-1 / 2 \int_{x_{3}=0} \tilde{P}_{i}^{*}\left[G_{i, l}\right] \tilde{P}_{l} d \mathbf{Q}
$$

The total energy is in fact given by the sum $F=F_{\text {chem }}+E_{\text {el }}$ (Eqs. (2.1) and (3.2)).

The $\mathbf{P}$ force is induced by the phases intrinsic stress misfit. Let note $\sigma_{A}^{0}$ and $\sigma_{B}^{0}$ the intrinsic stress tensor of the A and B phases, respectively. At position $\mathbf{r}$, the intrinsic stress is thus given by $\sigma^{0}(\mathbf{r})=\sigma_{B}^{0}+\delta \sigma^{0} \theta(\mathbf{r})$ where we introduce the tensor $\delta \sigma^{0}=\sigma_{A}^{0}-\sigma_{B}^{0}$. The induced force is simply obtained by deriving $\sigma^{0}$ with respect to the surface coordinates, it gives:

$$
P_{i}=\sum_{j=1,2} \delta \sigma_{i j}^{0} \frac{\partial \theta}{\partial x_{j}}
$$

Here we propose to distinguish whether there is a strong directional anisotropy such as for vicinal surface or no broken surface symmetry. In the former case, if a negligible stress variation is induced along a direction which angle with respect to [100] is noted $\alpha$, the $\mathbf{P}$ vector must be zero if $\theta$ gradient points this direction. The $\delta \sigma^{0}$ tensor coefficients are developed to satisfy this condition and one may write $\mathbf{P}$ as follows:

$$
P_{i}=\Lambda_{i}\left[\sin (\alpha) \frac{\partial \theta}{\partial x_{1}}-\cos (\alpha) \frac{\partial \theta}{\partial x_{2}}\right]
$$

where we introduce three constants $\left\{\Lambda_{i}\right\}$. We restrict our study to a uniaxial stress with only one non zero $\Lambda_{i}$ which is $\Lambda_{3}=\Lambda$. According to our tests, non zero $\Lambda_{1}$ and $\Lambda_{2}$ involve no qualitative change in our results.

If the surface square symmetry is preserved, the $\delta \sigma^{0}$ tensor is invariant under the square symmetry group operators. So $\delta \sigma_{12}^{0}=\delta \sigma_{21}^{0}, \delta \sigma_{31}^{0}=\delta \sigma_{32}^{0}$ and $\delta \sigma_{11}^{0}=\delta \sigma_{22}^{0}$. Again we introduce the $\Lambda$ parameter setting that $\delta \sigma_{11}^{0}=\delta \sigma_{22}^{0}=\Lambda$. The surface is assumed to be a perfect plane so the $P_{3}$ component is negligible, i.e., $\delta \sigma_{31}^{0}=\delta \sigma_{32}^{0}=0$. Non zero coefficients $\mu=\delta \sigma_{12}^{0}=\delta \sigma_{21}^{0}$ implies a shear that may be induced by the internal structures of the 
coexistent phases. To a first step, we choose to ignore such a possibility, i.e., $\mu<<\Lambda$. Thus the force $\mathbf{P}$ is simply proportional to the $\theta$ gradient.

The parameter $\Lambda$ is a constant that fixes the amplitude of the surface force and it may vary from an experimental case to another. In our model, for each different set of crystal elastic coefficients, $\Lambda$ is adjusted, i.e., our numerical calculations are performed with $\Lambda=40 \mathrm{~mJ} / \mathrm{m}^{2}$ (or $\Lambda=0.25 \mathrm{eV} / \AA^{2}$ ) for the Copper, Gold and Niobium crystal cases and $\Lambda=57 \mathrm{~mJ} / \mathrm{m}^{2}$ (or $\Lambda=0.35 \mathrm{eV} / \AA^{2}$ ) for the Chromium case.

\section{KINETICS OF PHASE SEPARATION}

To modelize the phase separation kinetics, we use a standard spinodal decomposition theory which is precisely described in Ref. [12] for the 3-dimensional case. Extension to the 2-dimensional systems is straighfoward. The time evolution of the conserved quantity $\theta(\mathbf{r})$ is then driven by the Cahn-Hilliard equation (also known as model B):

$$
\frac{\partial \theta(\mathrm{r}, t)}{\partial t}=M \triangle \frac{\delta F}{\delta \theta(\mathrm{r}, t)}+\epsilon(\mathrm{r}, t)
$$

where $\epsilon$ is a Langevin stochastic term which simulates thermal fluctuations as proposed by H.E. Cook in [13]:

$$
\begin{aligned}
<\epsilon(\mathrm{r}, t) & >=0 \\
<\epsilon(\mathrm{r}, t) \epsilon\left(\mathrm{r}^{\prime}, t^{\prime}\right)> & =-2 k_{B} T M \Delta \delta\left(\mathrm{r}-\mathrm{r}^{\prime}\right) \delta\left(t-t^{\prime}\right)
\end{aligned}
$$

In the previous Eq. 4.1, $M$ is a mobility constant which value may be related to the Fick diffusion constant $D_{\text {Fick }}$. As known, for a given chemical species the parameter $D_{\text {Fick }}$ strongly depends on both temperature and coverage (see Ref. [16]). For simplicity, we assum here that $D_{\text {Fick }} \approx 10^{-6} \mathrm{~cm}^{2} / \mathrm{s}$ (see [16]). Neglecting the elastic term $E_{\text {el }}$, a linear expansion of Eq. 4.1 around a uniform coverage $\theta(r)=0.5$ shows that the Eq. 4.1 is equivalent to a diffusion equation for short wavelength perturbations (see [12]). Then, we establish $M . F_{0} \cdot \frac{d^{2} \hat{f}}{d \theta^{2}}=D_{\text {Fick }}$ which fixes approximately the mobility since $\frac{d^{2} \hat{f}}{d \theta^{2}}\left(\theta_{0}=0.5\right)=16$, in the 
present case. As both $D_{\text {Fick }}$ and $\frac{d^{2} \hat{f}}{d \theta^{2}}$ strongly decrease with a decreasing $\theta_{0}$, the mobility variations with respect to the average coverage can not be estimated in the general context of our work. For simplicity, we choose to keep it constant, i.e., it is equal to the mobility estimated at half coverage.

Regarding the kinetics of the coarse-grained surface, it is simply derived from the space and time discretization of Eq. 4.1. The previous linear development of Eq. 4.1 is still valid and it comes that the diffusion constant $D_{\text {Fick }}$ is rescaled as $D_{F i c k} / d^{2}$ due to the discretized Laplacian operator. It corresponds to the intuitive idea according to which the larger the coarse-graining is, i.e., the larger is $d$, the slower each unit cell of the coarse-graining evolves and thus the slower is the global kinetics. One guesses that the larger is a B domain, the longer is the time needed to transform its whole area in a A phase region. Thus we are able to propose a rough estimation of the surface kinetics time scale. Our computer calculations work with a time unit which is equivalent to $\delta t=d^{2} / D_{\text {Fick }} \approx 10^{-8} s$. To perform the time integration of Eq. 4.1, we use a time increment of $\left(10^{-3} \times \delta t\right)$.

The Eq. (4.1) is integrated starting from a uniform initial state for which $\theta(\mathbf{r})=\theta_{0}$. Spontaneous formation of domains is observed for a coverage $\theta_{0}$ lying between $0.25<\theta_{0}<$ 0.75. This range is imposed by the classical spinodal region defined by $\partial^{2} \hat{f} / \partial^{2} \theta<0$ (see [12]). These limits are indeed slightly modified in presence of elastic interactions. The temperature dependency may be activated in the functional $F_{\text {chem }}$, in the diffusion coefficient $D_{\text {Fick }}$, and in the amplitude of the Langevin noise. The external parameters are the material elastic constants that fix $\chi$, the average coverage $\theta_{0}$, the surface stress $\Lambda$ and the $\alpha$ angle for both isotropic and anisotropic stress cases.

Our numerical findings confirm the analytical results of Refs. [1] [3]: the phase separation is frustrated by long range interaction due to the crystal surface stress, i.e., the kinetics yields a final steady state. As model input parameters, one may retain that the domain boundary energy is of order $I=10 \mathrm{meV} / \AA$ and the stress amplitude is either $\Lambda=40 \mathrm{~mJ} / \mathrm{m}^{2}$ (or $\Lambda=0.25 \mathrm{eV} / \stackrel{\circ}{A}^{2}$ ) for the Copper, Gold and Niobium crystal cases or $\Lambda=57 \mathrm{~mJ} / \mathrm{m}^{2}$ (or 
$\left.\Lambda=0.35 \mathrm{eV} / \AA^{2}\right)$ for the Chromium case.

In the case of a broken surface symmetry with uniaxial stress, the kinetics yields non branched stripes structures with spacial periodicity along a given direction as shown on Fig. $\square$ (for $\alpha=\pi / 4$ ). The selected period strongly decreases when $\Lambda$ increases but the formula proposed in Refs. [1]2] which gives the period versus the model parameters, has not been confirmed by our computations because it required many long time computer runs. It is the purpose of a forthcoming paper. For a coverage $\theta_{0}=0.3$ (see second row in Fig.(V), the time needed to reach the final stripe structure is longer than for $\theta_{0}=0.5$. As soon as the nucleation occures, it appears some distorted stripes length of which increases with coverage. During the coalescence regime, at $\theta_{0}=0.5$, the stripes branch to each other before their connections move along the stripe sides such that it eliminates the domain which separates the primary stripe couple. The mecanism is very different at low coverage (see second row of Fig.(V), since the short domains move to align themself with longer stripes.

For a preserved surface symmetry, the kinetics computer calculations results are pre-

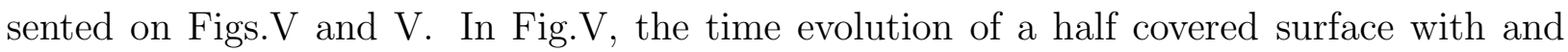
without induced stress is shown. One notes that for a negligible elastic interaction, i.e., $\Lambda=0$, the domains never stop growing because of the Ostwald's ripening [12, 18]. We had to stop the simulation when the size of the domains is of the order of the whole sample. For $\Lambda \neq 0$, the kinetics is radically different since the system is driven to a "self-organized" final state. Beyong an evolving stage which duration decreases as $\Lambda$ increases, the surface reaches a steady structure as shown by the two last pictures of the second and the third rows in Fig. $\nabla$. The specific size and the distance between the neighboring domains are determined by the value of $\Lambda$. The larger is $\Lambda$, the smaller are the domain sizes. According to our calculations, the final state is composed of branched stripes with tips as shown

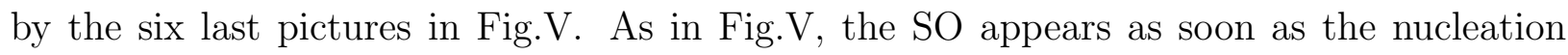
occures. At this stage, the domains already arrange along preferential orientations. The structure evolves such that the thinest branches disappear to the benefit of the thick stripes 
by first consuming the tips. The stripe orientations depend clearly on the sign of $\chi$ : for $\chi>0$ as for Chromium, stripes are along either [110] or [110] while for $\chi<0$ as for Copper, stripes are along either [100] or [010]. According to the few experimental results about SO of chemisorbed mono-layer, the adsorption of Nitrogen on a (001) Chromium surface has been analysed with STM by M. Pinczolits et al. in Ref. [19]. Infortunately, none of their results allows to conclude about the possible arrangement of Nitrogen atoms along [110] or [1]10] directions. The experimental results obtained by [6,7] about (001) Copper surface are in a good agreement with our calculations.

In Fig. $\mathrm{V}$, the plot of the ratio $\delta F /\left(F_{0} . S\right)$ where $S$ is the total surface size shows how the relative free energy $\Delta F$ evolves with time. This quantity $\delta F$ is the total free energy measured with respect to the free energy of a surface with only two compact domains of A and B phases. The stressed surface decomposition kinetics generates a steady state with lower energy because of a negative elastic energy $E_{e l}$. After a nucleation regime where the total energy decreases strongly as time increases, the energy reaches an asymptotic value. The asymptotic state free energy clearly depends on the coverage $\theta_{0}$ and the difference $\delta F\left(\theta_{0}=0.5\right)-\delta F\left(\theta_{0}=0.25\right)$ increases in absolute value with $\Lambda$. The same plot with no surface stress shows that the surface evolves in order to reduce its positive domain boundary energy. In such a case, the asymptotic energy difference of two surfaces with different coverages, i.e., $\delta F\left(\theta_{0}=0.5\right)-\delta F\left(\theta_{0}=0.25\right)$ becomes negligible.

At low coverage, as it was predicted in Ref. [3], an assembly of dots appear in the final stage. As for stripes, the arrangement of the dots also depends on the sign of $\chi$. The dots are arranged in an assembly of 1-dimensional rafts: the raft orientation is either along [110] or [110] for $\chi>0$ (first row of Fig. $\nabla$ ), and either along [100] or [010] for $\chi<0$ (second row of Fig. $(\nabla)$ ). In Fig. $\nabla$, the dots may be square shaped because of the relaxation of their elastic energy and the underlaying crystal symmetry. Comparison of the total free energy of a single A phase dot on a B surface, imposing different shapes to this dot, shows that the square shape is optimal, provided the area covered by the dot is larger than a critical size. For smaller sizes, the droplets are disk shaped. This critical size depends on both 
the elastic constants and $\Lambda$. As a consequence, in the first stage of the growth, when the domains have small area, all of them are disk shaped. In the case of Copper, before the surface reaches its steady state, most of the islands undergo a shape tranformation passing from a disk shape to a square shape (see the first row of Fig.Ш). In such a case, the domain size is clearly larger than the critical shape transformation size. In the left picture of the first row of Fig. $\rrbracket$, performed with Chromium elastic coefficients, it is the opposite case, since the disk shape domains never transform. In the right picture of the same row, increasing the surface coverage $\theta_{0}$ yields larger domains. Some of them overpasses the critical size to becomes square with [110] and [11̄0] oriented sides.

For a coverage lying between $\theta_{0}=0.25$ and half coverage, we find that the nanostructure of the final stage is composed of a mixing between short length stripes and dots (see Fig. $\nabla$ ). So, we deduce that the surface $\mathrm{SO}$ shows a crossover with respect to the coverage $\theta_{0}$, i.e., going from droplet structure at low coverage to a stripe structure at half coverage, passing through mixed structures.

The Fig. $\square$ shows the displacement field involved by the presence of the adsorbate. Nearby the surface, vortices are induced inside the bulk and extend over a depth of few ten nanometers. The maximum amplitude of the displacements is of few $0.1 \AA$. With both a molecular dynamics study and a linear elasticity analysis of the specific $\mathrm{N} / \mathrm{Cu}(001)$ case, those structures are also found by B. Croset et al. [17] who first pointed them to us. Moreother they showed that the bulk displacements due to vortices contribute to X-ray diffraction and they deduce a surface force amplitude measurement: $2.210^{-9} \mathrm{~N}^{-a t}{ }^{-} 1$ (or 0.3 $\left.\mathrm{eV} / \AA^{2}\right)$. In our model, the $\Lambda$ amplitude has same order of magnitude.

For (001) Copper surface case, an induced shear is tested, i.e., a non zero $\mu=\delta \sigma_{12}^{0}=\delta \sigma_{21}^{0}$ (defined above). Both the island shape and the arrangement directions are tilted with an angle that depends on the ratio $\mu / \Lambda$ (see Fig. $\nabla$ ).

The previous computations are performed with no thermal fluctuations. Putting on the Langevin noise in Eq. (4.1), with an amplitude which is related to a temperature by Eq. (4.3), we performed the same simulations and obtained similar final states with the same 
structures. This demonstrates the stability of both the kinetics and the final state. At half coverage and for the Copper elastic coefficients, the amplitude of the thermal noise may be increased up to the melting point of the Copper metal. Our method does not simulate the desorption process which could be activated at lower temperature than the crystal melting point. Moreother the white noise amplitude is calculated to satisfy the fluctuation dissipation theorem which is strictly valid around the thermodynamical equilibrium. Nevertheless the use of the Langevin noise allows to estimate qualitatively the surface structure ability to stand up to thermal fluctuations. Here the nanometric self-organised surface structure are proved to be very strong.

Similar results have been obtained with elastic constants of Gold $(\chi<0)$ and with Niobium elastic constants $(\chi>0)$ with a smaller amplitude $\Lambda$, indeed (see above for details).

\section{CONCLUSION AND PERSPECTIVES}

In the present paper, we investigated the patterns that are yielded from the (001) cubic crystal surface SO. If the surface square symmetry is preserved, the nanometric morphologies are proved to depend on both the coverage and the elastic coefficients of the crystal. One-dimentional rafts of dots (with disk shape or square shape) occur at low coverage and branched stripes appear at half coverage. Rafts and stripes are aligned with either [100] or [010] for a negative anisotropy factor and with either [110] or [1]̄0] for a positive anisotropy factor. Our computations have been performed with different cubic metal elastic coefficients, e.g., Gold, Copper, Chromium and Niobium. Obviously, it is possible to extend our calculations to the surfaces of either alloys or ionic crystal with cubic symmetry. In case of metal, this study should be extended to other surface symmetries which exibits interesting properties such as the (111) Gold surface e.g., the Gold Herringbone reconstruction (see, e.g., Refs. [4, 20]), and as the (110) Copper surface, e.g., the Oxyde adsorption (see Ref. [21]). Then, in the framework of the spinodal theory for SO, only the elastic Green function should be modified. 
In some specific cases, the crystal surface SO displays experimental features that are not included in the present model. The STM analysis of $\mathrm{N} / \mathrm{Cu}(001)$ performed by Ellmer et al. [7] demonstrates that the surface morphologies are not equivalent for coverage $\theta_{0}=(0.5+x)$ and for $\theta_{0}=(0.5-x)$, as one would expect from the spinodal decomposition theory we proposed. In addition, attractive interactions between Nitrogen islands seems to be dominant at very low coverage. We also note a remarkable result of T.W. Fishlock et al. [8] on $\mathrm{Br} / \mathrm{Cu}(001)$ where STM analysis shows bromine islands organized in a chessboard at half coverage while we would expect branched stripes. We believe that our simple method can be improved in order to investigate those specific cases even if it is clear that a continuous approach is limited to the mesoscopic scale analysis, e.g., it seems hopeless to include certain atomic scale features as surface dislocations, or ad-atom missing row.

In the present work, the chemisorbed layers thickness is neglected. As shown in Ref. 222 for the Silicon quantum dots, this thickness plays a role in the arrangement of dots but for the moment no idea emerges to tackle this problem on the basis of our approach.

For simplicity, we ignored the possible internal structures of each phases. If not it may involve both anisotropy and shear that are expected to modify the mesoscopic patterns. Moreother, some translational and orientational variants may be yielded from the different way the ad-atoms arrange on the underlying surface crystal lattice. Those variants can be treated in the present model by developing the free energy density with respect to the so called long range order parameters. This extension is well known within the phase field method for alloy physics [10]. As a consequence of the coexistent variants, some anti-phase boundaries should appear between domains and play a role in the pattern growth.

In summary, the model we present here is a promising start to describe and also to predict the nanostructures induced by self-organization of a crystal surface. 


\section{REFERENCES}

[1] V.I. Marchenko, Sov. Phys. JETP 54, 605 (1981).

[2] O.L. Alerhand, David Vanderbilt, R.D. Meade, and J.D. Joannopoulos, Phys. Rev. Lett. 61, 1973 (1988).

[3] Kwok-On Ng and David Vanderbilt, Phys. Rev. B 52, 2177 (1995).

[4] V. Repain, J-M. Berroir, B. Croset, and S. Rousset, Phys. Rev. Lett. 845367 (2000).

[5] S. Song, M. Yoon, S.G.J. Mochrie, G.B. Stephenson and S.T. Milner Surf. Sci. 37237 (1997).

[6] F. M. Leibsle, C. F. J. Flipse, and A. W. Robinson, Phys. Rev. B 47, 15865 (1993).

[7] H. Ellmer, V. Repain, S. Rousset, B. Croset, M. Sotto and Peter Zeppenfeld, submitted to Phys. Rev. Lett. (2001).

[8] T.W. Fishlock, J.B. Pethica and R.G. Egdell, Surf. Sci., 445, 47 (2000).

[9] M. Sotto, B. Croset, Surf. Sci. 461, 78 (2000).

[10] A.G. Khachaturyan, Theory of Structural Transformations in Solids, chap. 7, Ed. WileyInterscience, New-York (1983).

L.Q. Chen and A.G. Khachaturyan, Phys. Rev. Lett., 70, 1477 (1993).

Yunzhi Wang, L.Q. Chen and A.G. Khachaturyan, in Computer in Material Science, vol. 308 NATO ASI Series, Eds. H.O. Kirchner, L.P. Kubin and V. Pontikis (1996) p. 325.

[11] K. Kassner and C. Misbah, Europhys. Lett., 46, 217 (1999); A. Karma, Y. H. Lee and M. Plapp, Phys. Rev. E, 61, 3996 (2000).

[12] J.D. Gunton, M.M. San Miguel, and P.S. Sahni, Phase Transitions and Critical Phenomena, Academic Press, Vol. 8, Eds. C. Domb and J.L. Lebowitz, (1983) p. 269. 
[13] H.E. Cook, Acta Metallurgica, 18, 297 (1970).

[14] E.M. Lifshitz and L. D. Landau, Theory of Elasticity (Theoretical Physics, Vol.7), $3^{\text {rd }}$ edition Butterworth-Heinemann, (1995), pp. 54-55.

[15] J. Villain and A. Pimpinelli, Physique de la Croissance Cristalline, Appendice I, Ed. Eyrolles , Saclay (1995).

[16] A. Zangwill, Physics at surfaces, Cambridge University Press, (1988) p. 380.

[17] B. Croset et al. , submitted to Phys. Rev. Lett. (2001).

[18] I.M. Lifshitz and V.V. Slyozov, J. Phys. Chem. Solids, 19, 35 (1961);

C. Wagner, Z. Elektrochem., 65, 581 (1961).

[19] M. Pinczolits et al., Surf. Sci., 39678 (1998).

[20] S. Narasimhan and D. Vanderbilt, Phys. Rev. Lett., 69, 1564 (1992).

[21] Klaus Kern, Horst Niehus, Axel Schatz et al., Phys. Rev. Lett. 67, 855 (1991).

[22] J. Tersoff, C. Teichert and M. G. Lagally, Phys. Rev. Lett., 76, 1675 (1996). 


\section{Figure captions}

Fig. 1 (A-F): Phase separation kinetics of a $(001)$ cubic crystal surface $\left(\chi=\chi_{A u} \approx-0.5\right)$ with a stress directional anisotropy $\alpha=\pi / 4$ (defined in the text). Pictures occurence time is indicated at the low left hand corners: time unit is around $10 \mathrm{~ns}$. Kinetics is started at initial time $t=0$ with a uniform surface state. The direction [010] is indicated.

Fig. 2 (A-I): Phase separation kinetics on a (001) cubic crystal surface with negligible stress, i.e., $\Lambda=0$ (defined in the text) first row, and with $\Lambda=40 \mathrm{~mJ} / \mathrm{m}^{2} \chi=\chi_{C u}=-1$, second row and $\Lambda=57 \mathrm{~mJ} / \mathrm{m}^{2}$ and $\chi=\chi_{C r}=1$ third rows.

Fig. 3: Time evolution of the total free energy $\delta F$ for negligible stress, i.e., $\Lambda=0$, and for a stressed surface, i.e., $\Lambda=40 \mathrm{~mJ} / \mathrm{m}^{2}$ for different coverages: $\theta_{0}=0.25$ (dashed lines) and $\theta_{0}=0.5$ (solid lines). Time unit is $10 \mathrm{~ns}$ and the free energy is divided by the constant $F_{0} . S$ (defined in the text).

Fig. 4 (A-B) and 5 (A-B): First row: steady states of a (001) cubic crystal surface with $\Lambda=40 \mathrm{~mJ} / \mathrm{m}^{2}, \chi=\chi_{C u}=-1, \theta_{0}=0.21$ (on the left) and $\theta_{0}=0.35$ (on the right).

Second row: steady state of a (001) cubic crystal surface with $\Lambda=57 \mathrm{~mJ} / \mathrm{m}^{2}, \chi=\chi_{C r}=1$ , $\theta_{0}=0.21$ (on the left) and $\theta_{0}=0.35$ (on the right).

Fig. 6 : Profile of the displacement field inside the bulk, for $\Lambda=40 \mathrm{~mJ} / \mathrm{m}^{2}, \chi=\chi_{C u}=$ -1 and $\theta_{0}=0.21$. The arrows lenght is proportional to the displacement (multiplied by one hundred). A distance $d=1 \mathrm{~nm}$ separates neighboring sites of the coarse-grained lattice. The directions [010] and [001] are indicated.

Fig. 7: Steady state of a (001) cubic crystal surface with $\Lambda=40 \mathrm{~mJ} / \mathrm{m}^{2}, \chi=\chi_{C u}=-1$ , $\theta_{0}=0.3$ and a shear component $\delta \sigma_{12}^{0}=\delta \sigma_{12}^{0}=\Lambda / 2$. 
This figure "fig1A_proville.jpg" is available in "jpg" format from: http://arxiv.org/ps/cond-mat/0109237v1 
This figure "fig1B_proville.jpg" is available in "jpg" format from: http://arxiv.org/ps/cond-mat/0109237v1 
This figure "fig1C_proville.jpg" is available in "jpg" format from: http://arxiv.org/ps/cond-mat/0109237v1 
This figure "fig1D_proville.jpg" is available in "jpg" format from: http://arxiv.org/ps/cond-mat/0109237v1 
This figure "fig1E_proville.jpg" is available in "jpg" format from: http://arxiv.org/ps/cond-mat/0109237v1 
This figure "fig1F_proville.jpg" is available in "jpg" format from: http://arxiv.org/ps/cond-mat/0109237v1 
This figure "fig2A_proville.jpg" is available in "jpg" format from: http://arxiv.org/ps/cond-mat/0109237v1 
This figure "fig2B_proville.jpg" is available in "jpg" format from: http://arxiv.org/ps/cond-mat/0109237v1 
This figure "fig2C_proville.jpg" is available in "jpg" format from: http://arxiv.org/ps/cond-mat/0109237v1 
This figure "fig2D_proville.jpg" is available in "jpg" format from: http://arxiv.org/ps/cond-mat/0109237v1 
This figure "fig2E_proville.jpg" is available in "jpg" format from: http://arxiv.org/ps/cond-mat/0109237v1 
This figure "fig2F_proville.jpg" is available in "jpg" format from: http://arxiv.org/ps/cond-mat/0109237v1 
This figure "fig2G_proville.jpg" is available in "jpg" format from: http://arxiv.org/ps/cond-mat/0109237v1 
This figure "fig2H_proville.jpg" is available in "jpg" format from: http://arxiv.org/ps/cond-mat/0109237v1 
This figure "fig2I_proville.jpg" is available in "jpg" format from: http://arxiv.org/ps/cond-mat/0109237v1 


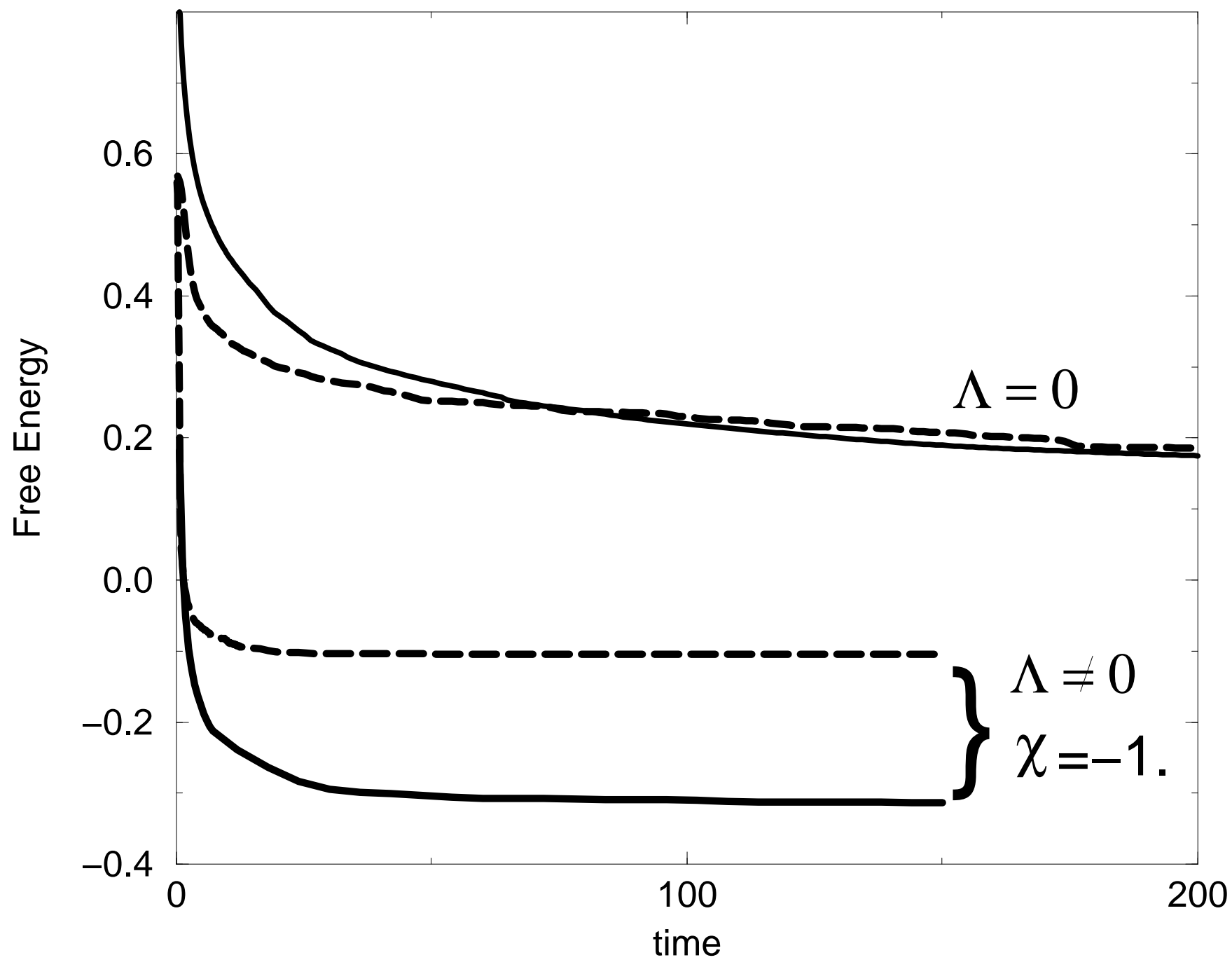


This figure "fig4A_proville.jpg" is available in "jpg" format from: http://arxiv.org/ps/cond-mat/0109237v1 
This figure "fig4B_proville.jpg" is available in "jpg" format from: http://arxiv.org/ps/cond-mat/0109237v1 
This figure "fig5A_proville.jpg" is available in "jpg" format from: http://arxiv.org/ps/cond-mat/0109237v1 
This figure "fig5B_proville.jpg" is available in "jpg" format from: http://arxiv.org/ps/cond-mat/0109237v1 


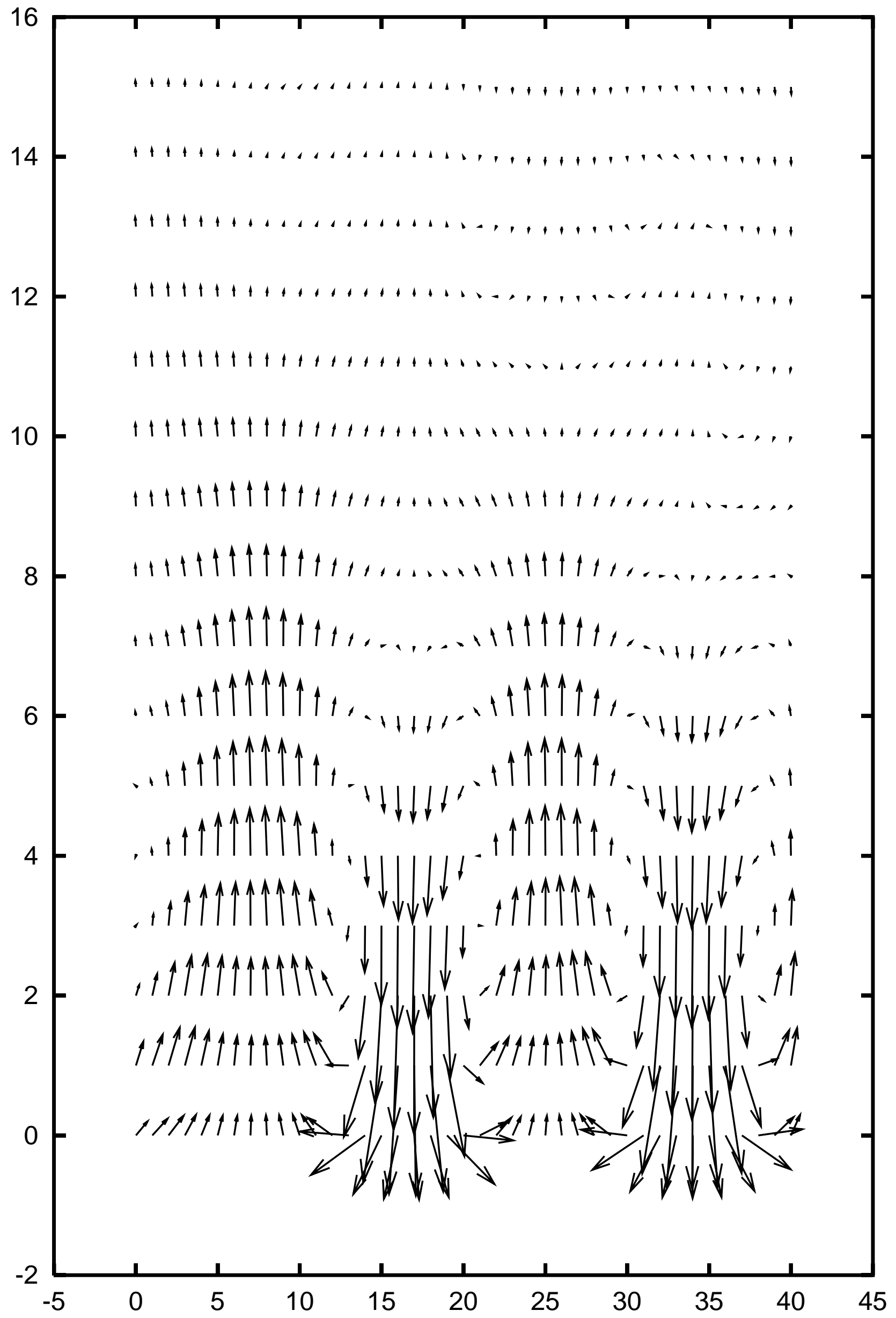


This figure "fig7_proville.jpg" is available in "jpg" format from: http://arxiv.org/ps/cond-mat/0109237v1 